\title{
Tunneling time through a rectangular barrier
}

\author{
Veríssimo M. de Aquino, ${ }^{1}$ Valdir C. Aguilera-Navarro, ${ }^{1,2}$ Mario Goto, ${ }^{1}$ and Hiromi Iwamoto ${ }^{1}$ \\ ${ }^{1}$ Departamento de Física/CCE, Universidade Estadual de Londrina, 86051-970 Londrina, PR, Brazil \\ ${ }^{2}$ Instituto de Física Teórica, Universidade Estadual Paulista, 01405-900 São Paulo, SP, Brazil
}

(Received 1 June 1998)

\begin{abstract}
In this paper, we discuss the tunneling time of a quantum particle through a rectangular barrier. The reflection and transmission times associated with the wave packets representing the particle are discussed. By using an initial Gaussian momentum distribution, we carry out a comparative analysis of the stationary phases of the incident, reflected, and transmitted wave packets leading to the reflection and transmission times $\Delta t_{R}$ and $\Delta t_{T}$, respectively. In the present treatment of this old and very known problem we take into account the deformations of the reflected and transmitted momentum distributions. These deformations produce a dependence of the reflection and transmission times on the location of the initial wave packet. In a parallel calculation, by numerically monitoring the time evolution of the system, we characterize a reflection and a transmission time. Such times agree with the ones obtained via the stationary phase method.
\end{abstract}

[S1050-2947(98)07912-8]

PACS number(s): 03.65.-w, 73.40.Gk, 73.30.+y, 73.40.-c

\section{INTRODUCTION}

In the literature, we can find a number of papers suggesting different expressions for the so-called tunneling time, the time a quantum particle spends in a classically forbidden potential region. In particular, we cite some of those works that propose a method of associating the tunneling time with a phase time $\Delta \tau_{\varphi}[1-6]$. According to such a method, the tunneling time is obtained from an analysis of the phase of the function that describes a wave packet incident on the potential.

In Ref. [3], Büttiker and Landauer point out some difficulties in the method of phase due to the deformation of the wave packet during its time evolution. Hauge et al. [4] are concerned with the lack of clarity in dealing with situations in which the momentum distribution associated with the wave packet has finite width. In Ref. [5], a correction to the method is discussed and the tunneling time obtained in firstorder approximation coincides with the phase time. Collins [6] presents some numerical results to establish validity limits to the phase method.

In this paper, we consider a quantum particle of energy $E$ incident on a rectangular barrier potential of height $V_{0}>E$. The particle is initially represented by a Gaussian distribution and the deformations in the momentum distribution of the reflected and transmitted wave packets are taken into account. The reflection and transmission time are obtained using the stationary phase method [7]. The validity of the expression so obtained for $\Delta \tau_{\varphi}$ is discussed. An extensive numerical analysis is made in order to support the results.

In Sec. II, we formulate the quantum problem and present its (well known) solution in order to define notation. The wave packets are constructed and discussed in Sec. III. Their time evolutions are studied in Sec. IV. Finally, in Sec. V we develop an extensive numerical analysis and present the results.

\section{THE PROBLEM AND ITS SOLUTION}

We consider a particle of mass $m$ and energy $E$ moving towards a rectangular barrier $V(x)$ defined by

$$
V(x)=\left\{\begin{array}{l}
0, x<0, \text { region } 1, \\
V_{0}, 0<x<a, \text { region } 2, \\
0, x>a, \text { region } 3 .
\end{array}\right.
$$

The corresponding Schrödinger equation and its solution are well known and presented in practically all quantum mechanics text books. For the sake of notation, we present in this section a summary of the relevant results. $[2,8]$

The energy eigenfunction $u_{E}(x)$, for $E<V_{0}$, is given by

$$
u_{E}(x)=\left\{\begin{array}{l}
u_{1}(x)=A e^{i k x}+A^{\prime} e^{-i k x}, \quad \text { region } 1, \\
u_{2}(x)=B e^{-\rho x}+D e^{\rho x}, \quad \text { region } 2, \\
u_{3}(x)=C e^{i k x}, \quad \text { region } 3,
\end{array}\right.
$$

with

$$
k^{2}=\frac{2 m E}{\hbar^{2}}, \quad k_{0}^{2}=\frac{2 m V_{0}}{\hbar^{2}}, \quad \rho^{2}=k_{0}^{2}-k^{2} .
$$

The boundary conditions at $x=0$ and $x=a$ determine the coefficients in Eq. (2), which can be given in a convenient notation if we introduce the quantities $\theta, R$, and $\alpha$ through the definitions

$$
\begin{aligned}
& \cos \theta=\frac{k}{k_{0}} \quad \text { and } \sin \theta=\frac{\rho}{k_{0}}, \\
& \left(e^{2 i \theta} e^{-2 \rho a}-e^{-2 i \theta}\right)=R e^{-i \alpha},
\end{aligned}
$$

hence

$$
R=\sqrt{1+e^{-4 \rho a}-2 e^{-2 \rho a} \cos 4 \theta}
$$

and

$$
\tan \alpha=\operatorname{coth} \rho a \tan 2 \theta .
$$

In terms of these quantities, we have that 


$$
\begin{gathered}
A=\frac{\left(e^{-2 \rho a}-1\right)}{R} e^{-i \alpha}, \\
B=-\frac{2 \cos \theta}{R} e^{i \theta} e^{-i \alpha}, \\
C=-e^{-\rho a} \frac{2 i \sin 2 \theta}{R} e^{-i k a} e^{-i \alpha},
\end{gathered}
$$

and

$$
D=e^{-2 \rho a} \frac{2 \cos \theta}{R} e^{-i \theta} e^{-i \alpha} .
$$

As a check, it can be easily seen that the reflection coefficient $|A|^{2}$ and the transmission coefficient $|C|^{2}$ satisfy the expected relation

$$
|A|^{2}+|C|^{2}=1
$$

General solutions to the Schrödinger equation can be obtained as superposition of the eigenfunctions $u_{E}(x)$,

$$
\psi(x, t)=\int_{E} C_{E} e^{-(i / \hbar) E t} u_{E}(x) d E .
$$

Such superpositions define wave packets and are shaped according to the initial conditions of the physical system. In the next section we construct some of these wave packets, required for our present purpose.

\section{CONSTRUCTION OF THE WAVE PACKETS}

We start by constructing a wave packet that represents a free particle, with defined energy $E<V_{0}$, moving in region 1, defined in Eq. (1), towards the potential barrier. Classically, such a particle should be bounced by the barrier, but quantum mechanically there is a nonvanishing probability that the particle penetrates the barrier and emerges on the other side, again as a free particle. In terms of wave packets, part of the packet is reflected and part penetrates the potential region and emerges on the other side as another wave packet.

As initial conditions, let us place the incident particle somewhere to the left of the barrier, specifically at the position $\bar{x}=-\ell$, and let it move to the right with group velocity $\bar{v}$. The best we can do to represent this particle, from the point of view of quantum mechanics, is to consider a very narrow wave packet with a sharp peak in the momentum probability distribution, $|\phi(k)|^{2}$, as well as in the spatial probability distribution, $|\psi(x)|^{2}$, around the respective mean values

$$
\bar{k}=\frac{m \bar{v}}{\hbar}
$$

and $\bar{x}=-\ell$ at $t=0$. Such a wave packet will arrive at the barrier, in $x=0$, at time

$$
t_{0}=\ell / \bar{v}=m \ell / \hbar \bar{k}
$$

Once the initial wave packet $\psi(x)=\psi(x, 0)$ is defined, we can obtain the momentum distribution by imposing

$$
\psi(x)=\psi(x, 0)=\frac{1}{\sqrt{2 \pi}} \int_{0}^{k_{0}} d k \phi(k) e^{i k x}
$$

so that

$$
\phi(k)=\frac{1}{\sqrt{2 \pi}} \int_{-\infty}^{\infty} e^{-i k x} \psi(x) d x .
$$

The integral in $k$ must be done through $k_{0}$ since we are considering particles with $E<V_{0}$.

For practical purposes, we consider a Gaussian wave packet centered in $x=-\ell$, namely,

$$
\psi(x)=\left(\frac{2}{\pi x_{0}^{2}}\right)^{1 / 4} e^{i \bar{k}(x+l)} e^{-(x+l)^{2} / x_{0}^{2}}
$$

which corresponds to the momentum distribution centered in $\bar{k}$,

$$
g(k-\bar{k}) e^{i k l}=\frac{1}{\left(2 \pi \sigma_{k}^{2}\right)^{1 / 4}} e^{i k l} e^{-(k-\bar{k})^{2} / 4 \sigma_{k}^{2}}
$$

with

$$
\sigma_{k}^{2}=\left\langle k^{2}\right\rangle-\langle k\rangle^{2}
$$

We choose this momentum distribution for our wave packet imposing, however, the same previous upper limit to the momentum integration, i.e.,

$$
\psi(x, 0)=\frac{1}{\sqrt{2 \pi}} \int_{0}^{k_{0}} g(k-\bar{k}) e^{i k l} e^{i k x} d k
$$

Due to this restriction of $k$ to the region below $k_{0}$, Eq. (20) does not result exactly in the Gaussian spatial distribution Eq. (17). Nevertheless, it represents, with good accuracy a Gaussian wave packet centered in $x=-\ell$, at $t=0$, if the momentum distribution width (19) is taken sufficiently small such that the momentum distribution $g(k)$ goes quickly to zero as $k$ approaches $k_{0}$. So

$$
\int_{0}^{k_{0}} g(k-\bar{k}) e^{i k l} e^{i k x} d k \simeq \int_{-\infty}^{\infty} g(k-\bar{k}) e^{i k l} e^{i k x} d k .
$$

The above-mentioned condition is also necessary to assure that the momentum expectation value $\langle k\rangle$ is equal to the momentum distribution peak $\bar{k}$, i.e.,

$$
\langle k\rangle=\int_{0}^{k_{0}} k|g(k-\bar{k})|^{2} d k \simeq \int_{0}^{\infty} k|g(k-\bar{k})|^{2} d k=\bar{k} .
$$

Now we proceed to construct the wave packets in all three regions defined in Eq. (1). At any time, the wave function is given by 


$$
\psi(x, t)=\left\{\begin{array}{l}
\psi_{1}(x, t)=\psi_{I}(x, t)+\psi_{R}(x, t), \quad x<0, \\
\psi_{2}(x, t), \quad 0<x<a \\
\psi_{3}(x, t)=\psi_{T}(x, t), \quad x>a
\end{array}\right.
$$

where the indices $I, R$, and $T$ stand for incident, reflected, and transmitted, respectively. For the incident wave packet $\psi_{I}(x, t)$, we take a Gaussian probability distribution initially centered on $x=-\ell$, i.e.,

$$
\psi_{I}(x, t)=\frac{1}{\sqrt{2 \pi}} \int_{0}^{k_{0}} g(k-\bar{k}) e^{i k(x+l)} e^{-i \omega(k) t} d k,
$$

where $g$ is given by Eq. (18) and

$$
\omega=\omega(k)=\frac{\hbar k^{2}}{2 m} .
$$

The reflected wave packet is given by

$$
\begin{aligned}
\psi_{R}(x, t)= & \frac{1}{\sqrt{2 \pi}} \int_{0}^{k_{0}} g(k-\bar{k}) \frac{\left(e^{-2 \rho a}-1\right)}{R} \\
& \times e^{-i k(x-l)} e^{-i \omega(k) t} e^{-i \alpha} d k \\
= & \frac{1}{\sqrt{2 \pi}} \int_{0}^{k_{0}} \phi_{R}(k) e^{-i k(x-l)} e^{-i \omega(k) t} e^{-i \alpha} d k,
\end{aligned}
$$

where $\rho, \theta, R$, and $\alpha$ are defined in Eqs. (3), (4), (6), and (7), respectively, and

$$
\phi_{R}(k)=g(k-\bar{k}) \frac{\left(e^{-2 \rho a}-1\right)}{R}=|A| g(k-\bar{k}),
$$

where the reflection coefficient $A$ is given in Eq. (8).

Figure 1 displays the momentum distributions associated with the incident and reflected wave packets. It shows that the reflected wave packet presents a small shift to the left with respect to the incident one. This fact produces a shift in the average momentum associated with the distributions

$$
\langle k\rangle_{R}=\int_{0}^{k_{0}} k\left|\phi_{R}(k)\right|^{2} d k<\bar{k} .
$$

Since the reflected peak $\bar{k}_{R}$ is also shifted, it is expected that

$$
\langle k\rangle_{R}=\bar{k}_{R}<\bar{k} .
$$

The wave packet in the classically forbidden region is given by

$$
\begin{aligned}
\psi_{2}(x, t)= & \frac{1}{\sqrt{2 \pi}} \int_{0}^{k_{0}} g(k-\bar{k}) \frac{2 \cos \theta}{R} \bar{R} \\
& \times e^{-i \gamma(k, x)} e^{i k l} e^{-i \omega(k) t} e^{-i \alpha} d k \\
= & \frac{1}{\sqrt{2 \pi}} \int_{0}^{k_{0}} \phi_{2}(k, x) e^{-i \gamma(k, x)} e^{i k l} e^{-i \omega(k) t} e^{-i \alpha} d k,
\end{aligned}
$$

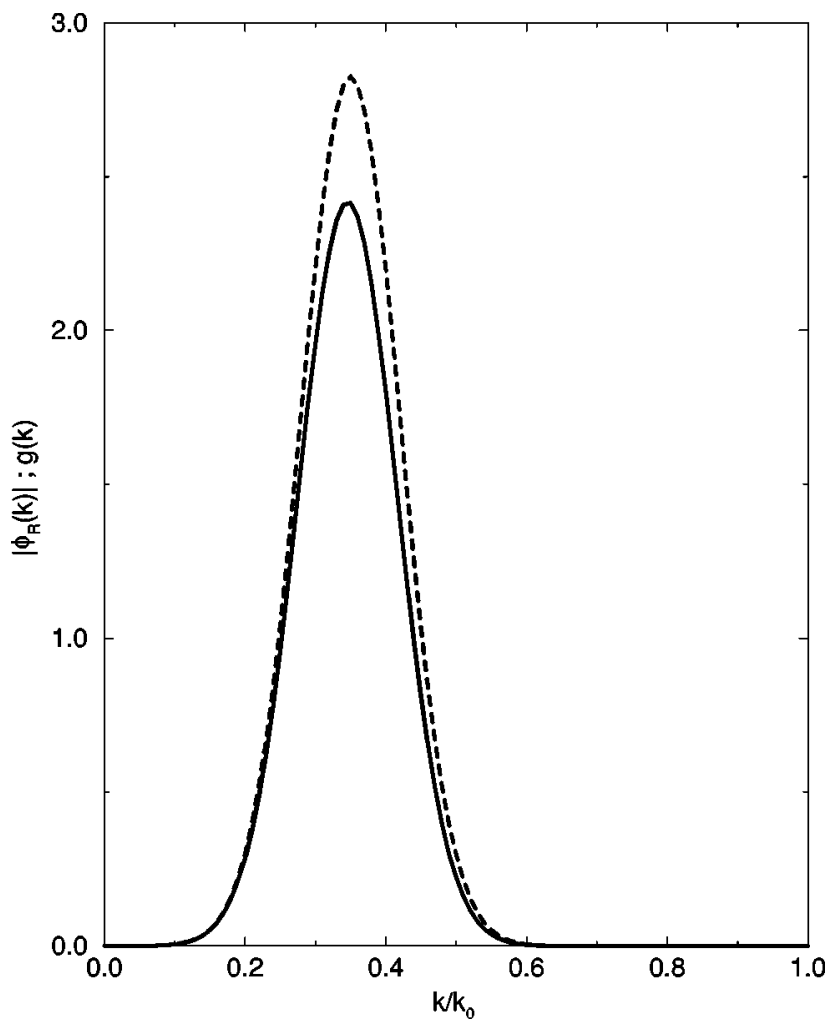

FIG. 1. Momentum distribution $\left|\phi_{R}(k)\right|$ (full line) and $g(k)$ (dashed line) for $\bar{k}=0.35 k_{0}, \sigma_{k}^{2}=0.0025 k_{0}^{2}$, and $k_{0} a=1$.

with

$$
\begin{gathered}
\phi_{2}(k, x)=g\left(k-\bar{k}_{0}\right) \frac{2 \cos \theta}{R} \bar{R}, \\
\bar{R}=\sqrt{e^{-4 \rho a} e^{2 \rho x}+e^{-2 \rho x}-2 e^{-2 \rho a} \cos 2 \theta},
\end{gathered}
$$

and

$$
\tan \alpha=\operatorname{coth}[\rho(x-a)] \tan 2 \theta .
$$

It should be noticed the $x$ dependence of the probability distribution $\phi_{2}$.

Finally, the transmitted wave packet is given by

$$
\begin{aligned}
\psi_{T}(x, t)= & \frac{1}{\sqrt{2 \pi}} \int_{0}^{k_{0}} g(k-\bar{k}) e^{-\rho a} \frac{2 \sin 2 \theta}{r} \\
& \times e^{i k(x+l-a)} e^{-i \omega(k) t} e^{-i \alpha} e^{-i \pi / 2} d k \\
= & \frac{1}{\sqrt{2 \pi}} \int_{0}^{k_{0}} \phi_{T}(k) e^{i k(x+l-a)} e^{-i \omega(k) t} e^{-i \alpha} e^{-i \pi / 2} d k,
\end{aligned}
$$

where

$$
\phi_{T}(k)=g(k-\bar{k}) e^{-\rho a} \frac{2 \sin 2 \theta}{R} .
$$




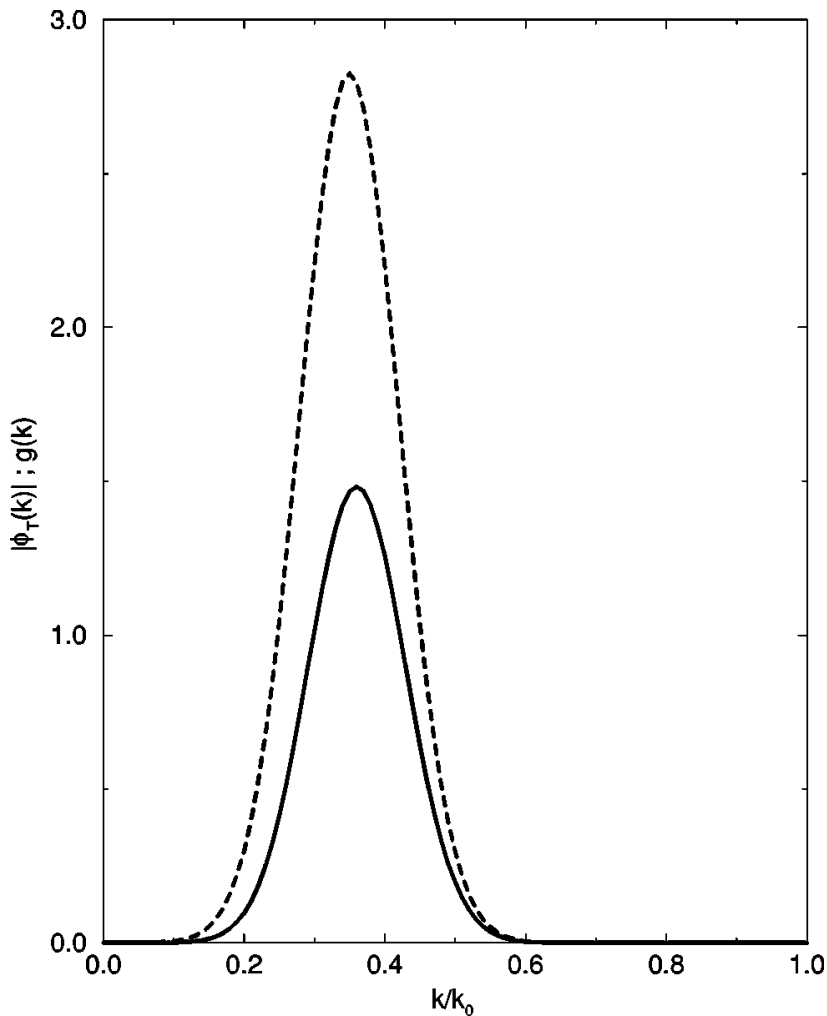

FIG. 2. Momentum distribution $\phi_{T}(k)$ (full line) and $g(k)$ (dashed line) for $\bar{k}=0.35 k_{0}, \sigma_{k}^{2}=0.0025 k_{0}^{2}$, and $k_{0} a=1$.

Figure 2 displays the momentum distribution associated with the transmitted wave packet. It shows that the transmitted wave is well localized around a well defined peak $\bar{k}_{T}$, which validates the stationary phase analysis to be carried out below. Figure 2 also shows that the transmitted momentum distribution is shifted to the right with respect to the initial distribution. Consequently, there is a shift in the average momentum of the transmitted wave with respect to the average value of the incident momentum, i.e.,

$$
\langle k\rangle_{T}=\int_{0}^{k_{0}} k\left|\phi_{T}(k)\right|^{2} d k>\bar{k} .
$$

A numerical analysis of the transmitted momentum distribution shows that the average value coincides with the peak value, i.e.,

$$
\langle k\rangle_{T}=\bar{k}_{T}>\bar{k}
$$

Table I contains the values of $\bar{k}_{T}$ for several values of $\bar{k}_{0}$. It can be seen that the peak deviation is greater for small values of $\bar{k}$ and decreases as $\bar{k}$ increases. There is practically no deviation for $\bar{k}>0.6$. Table II contains the values of $\bar{k}_{R}$ for several values of $\bar{k}$. It can be seen that the peak deviation is greater for great values of $\bar{k}$ and decreases as $\bar{k}$ decreases. There is practically no deviation for $\bar{k}<0.5 k_{0}$. Figure 3 displays the momentum distributions peaks of the transmitted and reflected wave packets and the respective shifts, for some values of $\bar{k}$.
TABLE I. $\bar{k}_{T}$ as a function of $\bar{k}$ for $\sigma_{k}^{2}=0.0025 k_{0}^{2}$ and $k_{0} a=1$.

\begin{tabular}{cccccc}
\hline \hline $\bar{k}$ & $\bar{k}_{T}$ & $\bar{k}$ & $\bar{k}_{T}$ & $\bar{k}$ & $\bar{k}_{T}$ \\
\hline 0.30 & 0.313 & 0.46 & 0.467 & 0.62 & 0.624 \\
0.32 & 0.332 & 0.48 & 0.486 & 0.64 & 0.644 \\
0.34 & 0.351 & 0.50 & 0.506 & 0.66 & 0.663 \\
0.36 & 0.370 & 0.52 & 0.526 & 0.68 & 0.683 \\
0.38 & 0.389 & 0.54 & 0.545 & 0.70 & 0.703 \\
0.40 & 0.409 & 0.56 & 0.565 & 0.72 & 0.723 \\
0.42 & 0.428 & 0.58 & 0.585 & 0.74 & 0.743 \\
\hline \hline
\end{tabular}

\section{EVOLUTION OF THE WAVE PACKETS}

\section{A. The incident wave packet}

As we have mentioned before, the maximum of the probability distribution $\left|\phi_{I}(k)\right|^{2}$, associated with the incoming particle, arrives at the barrier $(x=0)$ at time $t_{0}$ given by Eq. (14). The phase of the incident wave packets (24) is given by

$$
f_{I}(k)=(x+\ell) k-\omega(k) t .
$$

By imposing a stationary phase through the condition [7]

$$
\left.\frac{d f_{I}(k)}{d k}\right|_{k=\bar{k}}=0,
$$

we get the equation corresponding to the classical path in region 1 ,

$$
x(t)=\frac{\hbar \bar{k}}{m} t-\ell .
$$

As expected, this is the motion equation for a classical particle moving from $x=-\ell$, at $t=0$, to the right with velocity $\bar{v}=\hbar \bar{k} / m$, reaching the position $x=0$ at time $t=m \ell / \hbar \bar{k}$.

\section{B. The reflected wave packet}

The condition of stationary phase applied to the reflected wave packet (26) gives us

$$
x(t)=\ell-v_{R} t-\left.\frac{d \alpha(k)}{d k}\right|_{k=\bar{k}_{R}},
$$

TABLE II. $\bar{k}_{R}$ as a function of $\bar{k}$ for $\sigma_{k}^{2}=0.0025 k_{0}^{2}$ and $k_{0} a=1$.

\begin{tabular}{cccccc}
\hline \hline $\bar{k}$ & $\bar{k}_{R}$ & $\bar{k}$ & $\bar{k}_{R}$ & $\bar{k}$ & $\bar{k}_{R}$ \\
\hline 0.30 & 0.296 & 0.46 & 0.455 & 0.62 & 0.615 \\
0.32 & 0.16 & 0.48 & 0.475 & 0.64 & 0.635 \\
0.34 & 0.336 & 0.50 & 0.495 & 0.66 & 0.655 \\
0.36 & 0.356 & 0.52 & 0.515 & 0.68 & 0.675 \\
0.38 & 0.376 & 0.54 & 0.535 & 0.70 & 0.695 \\
0.40 & 0.396 & 0.56 & 0.555 & 0.72 & 0.715 \\
0.42 & 0.416 & 0.58 & 0.575 & 0.74 & 0.735 \\
\hline \hline
\end{tabular}




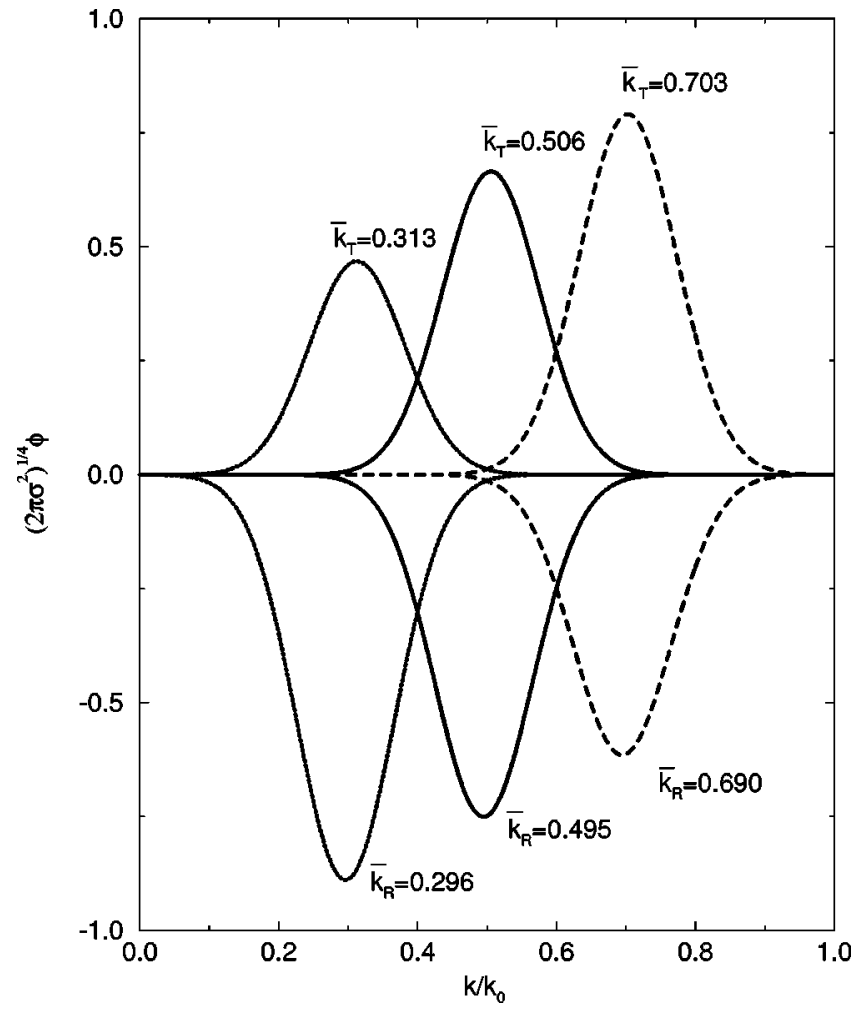

FIG. 3. Momentum distribution $\phi_{T}(k)$ and $\phi_{R}(k)$ for $\bar{k}=0.3 k_{0}$ (dotted line), $0.5 k_{0}$ (full line), $0.7 k_{0}$ (dashed line), $\sigma_{k}^{2}=0.0025 k_{0}^{2}$, and $k_{0} a=1$. Also displayed are the respective shifts corresponding to $\bar{k}_{T}$ and $\bar{k}_{R}$.

where

$$
v_{R}=\frac{\hbar \bar{k}_{R}}{m}
$$

and $\bar{k}_{R}$ is the value of $k$ that maximizes the distribution probability (27).

By imposing $x(t)<0$, the appropriate condition for the reflected wave packet (26), we observe, from the equation of motion (41), that the reflected wave packet presents a maximum that emerges from $x=0$ at time $t_{R}$ given by

$$
t_{R}=\frac{\ell-\bar{\alpha}_{R}}{v_{R}}
$$

with

$$
\bar{\alpha}_{R}=\left.\frac{d \alpha(k)}{d k}\right|_{k=\bar{k}_{R}}=-\left\{\frac{1}{\rho} \frac{(2 \sinh 2 \rho a-k a \sin 4 \theta)}{(\cosh 2 \rho a-\cos 4 \theta)}\right\}_{k=\bar{k}_{R}} .
$$

The time difference

$$
\Delta t_{R}=t_{R}-t_{0}=\frac{\ell}{v_{R}}-\frac{\bar{\alpha}_{R}}{v_{R}}-\frac{\ell}{\bar{v}}
$$

shows that the arrival at $x=0$ of the incident-wave-packet peak does not coincide with the departure of the reflectedwave-packet peak from $x=0$. As, in general, $v_{R}<\bar{v}$, the time

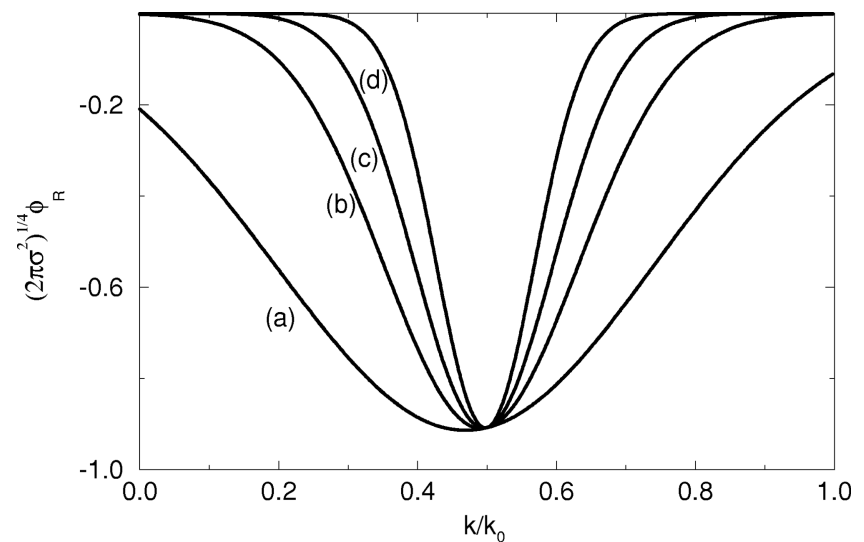

FIG. 4. Behavior of $\phi_{R}(k)$ as a function of the momentum $k$, for several distribution widths: $\sigma^{2}(k)=0.0250$ (a), 0.0100 (b), 0.0050 (c), and 0.0025 (d) in units of $k_{0}^{2}$.

interval $\Delta t_{R}$ is always positive, i.e., the peak of the reflected wave packet leaves the barrier after a delay time $\Delta t_{R}$ since the arrival of the incident packet.

As can be seen in Fig. 1, $\phi_{R}(k)$ has a maximum shifted to the left relative to the maximum of the distribution $g(k$ $-\bar{k})$. In Fig. 4, we observe that $\bar{k}_{R}$ goes to $\bar{k}$ when $g(k$ $-\bar{k})$ approaches a delta function $\delta(k-\bar{k})$, i.e.,

$$
\lim _{g(k-\bar{k}) \rightarrow \delta(k-\bar{k})} \bar{k}_{R}=\bar{k} .
$$

In this case, $v_{R} \rightarrow \bar{v}$ and the reflection time $\Delta t_{R}$, Eq. (45), goes to the so-called tunneling time obtained through the method of phases

$$
\Delta t_{R}=t_{R}-t_{0}=-\frac{\bar{\alpha}_{0}}{\bar{v}}=\Delta \tau_{\varphi}
$$

where

$$
\bar{\alpha}_{0}=\left.\frac{d \alpha(k)}{d k}\right|_{k=\bar{k}}
$$

\section{The transmitted wave packet}

For the transmitted wave packet $\psi_{T}(x, t)$ given in Eq. (34), the equation of motion obtained by using the stationary phase method is

$$
x+\ell-a-v_{T} t-\bar{\alpha}_{T}=0,
$$

where

$$
v_{T}=\frac{\hbar \bar{k}_{T}}{m}
$$

and $\bar{\alpha}_{T}$ is given by Eq. (44), but now evaluated at $\bar{k}_{T}$ instead of $\bar{k}_{R}$. The quantity $\bar{k}_{T}$ determines the maximum of the momentum distribution $\phi_{T}(k)$ given in Eq. (35). Obviously, in general, $\bar{k}_{T} \neq \bar{k}_{R}$ which implies different group velocities for the transmitted and reflected packets. 


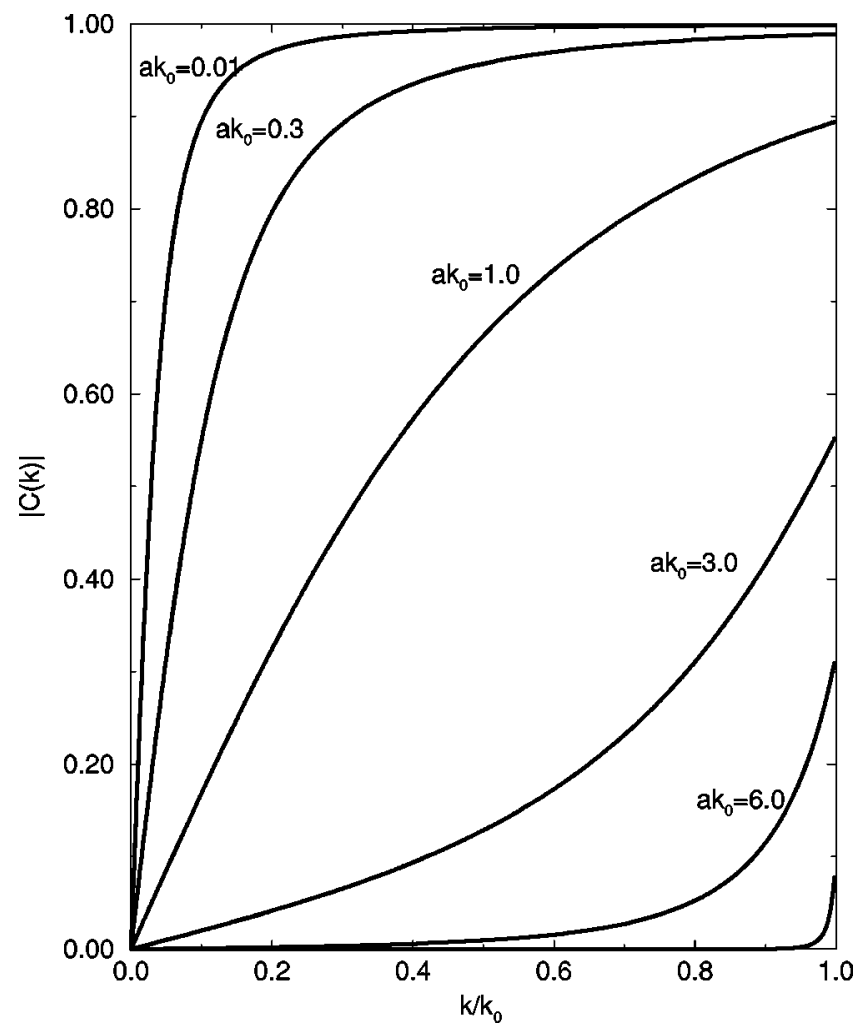

FIG. 5. Behavior of the transmission coefficient as a function of $k$ and the barrier width.

By imposing $x(t)>a$, the appropriate condition for the transmitted wave packet, a stationary phase for $\psi(x, t)$ will exist starting at time $t_{T}$ given by

$$
t_{T}=\frac{\ell}{v_{T}}-\frac{\bar{\alpha}_{T}}{v_{T}} .
$$

The time difference

$$
\Delta t_{T}=t_{T}-t_{0}=\frac{\ell}{v_{T}}-\frac{\bar{\alpha}_{T}}{v_{T}}-\frac{\ell}{\bar{v}}
$$

corresponds to the time interval between the arrival of the incident-wave-packet peak at $x=0$ and the departure of the transmitted-wave-packet peak from $x=a$.

Since $\bar{\alpha}_{T}>0$ and, in general, $v_{T}>\bar{v}$, the time interval $\Delta t_{T}$ may assume negative values meaning that the transmittedwave-packet peak emerges before the arrival of the incidentwave-packet peak. This phenomenon can occur because the faster components of the incident packet are more likely to tunnel through the barrier than the slower ones which are more likely to be bounced. This fact is illustrated in Fig. 5, which displays the transmission coefficient in function of the momentum $k$ for several values of the barrier width. We can see that for barriers with very small width, the transmission coefficient increases quickly to values near 1 . Distributions $g(k-\bar{k})$ not centered near $k=0$ generate transmitted distributions with peaks very close to those of $g(k-\bar{k})$. For large $k_{0} a$, the transmission coefficient is nearly zero in almost the whole range $0<k<k_{0}$. Only those distributions $g(k-\bar{k})$ centered very near $\bar{k}$ will present maxima of $\phi_{T}(k)$ shifted

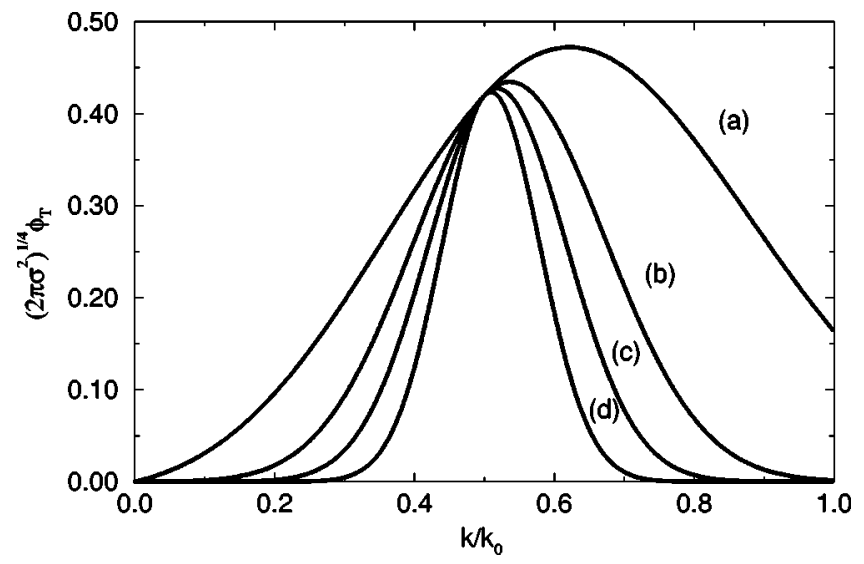

FIG. 6. Behavior of $\phi_{T}(k)$ as a function of the momentum $k$, for several distribution widths: $\sigma^{2}(k)=0.0250$ (a), 0.0100 (b), 0.0050 (c), and 0.0025 (d) in units of $k_{0}^{2}$.

relatively to the maximum of $g(k-\bar{k})$. This shift of $\bar{k}_{T}$ with respect to $\bar{k}$ increases for distributions centered around small values of $k$. For large values of $k_{0} a$, this effect is more evident for distributions centered on greater values of $k$. In general, $\phi_{T}(k)$ tends to have maximum shifted to the right with respect to the maximum of $g(k-\bar{k})$ and this shift depends on $\bar{k}$ as well as on the width $\Delta k$ of the distribution. When $g(k-\bar{k})$ approaches a delta distribution $\delta(k-\bar{k})$, $\phi_{T}(k)$ tends to present a maximum very close to the maximum of $g(k-\bar{k})$. An analogous analysis of the reflection coefficient and of the function $\phi_{R}(k)$ leads to similar results.

In Fig. 6, we observe that $\bar{k}_{T}$ goes to $\bar{k}$ as $g(k-\bar{k})$ approaches a delta function, i.e.,

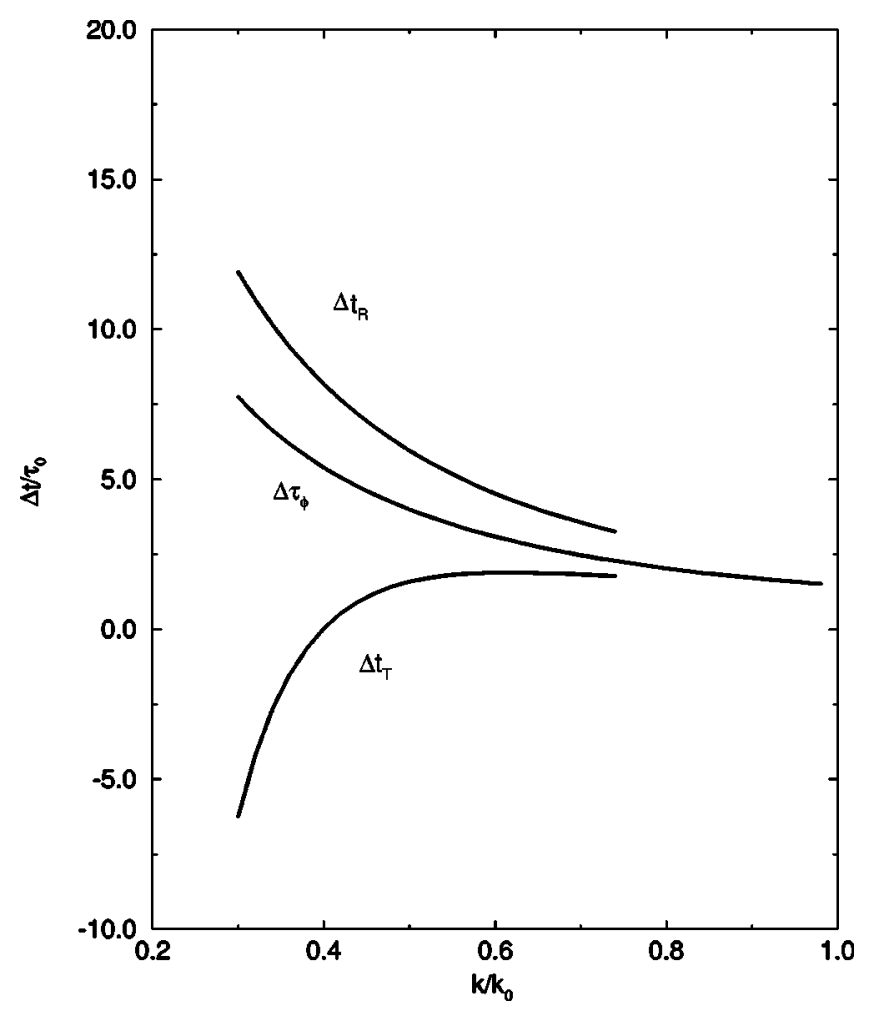

FIG. 7. Behavior of $\Delta t_{T}, \Delta t_{R}$, and $\Delta \tau_{\varphi}$ as a function of $k$ for $\sigma_{k}^{2}=0.0025 k_{0}^{2}, k_{0} a=1$, and $\ell / a=100$. 


$$
\lim _{g(k-\bar{k}) \rightarrow \delta(k-\bar{k})} \bar{k}_{T}=\bar{k} .
$$

In this case, $v_{T} \rightarrow \bar{v}$ and $\bar{\alpha}_{T} \rightarrow \bar{\alpha}_{0}$, and the transmission time $\Delta t_{T}$, Eq. (52), goes to $-\bar{\alpha}_{0} / \bar{v}$, i.e.,

$$
\Delta t_{T}=t_{T}-t_{0} \rightarrow-\frac{\bar{\alpha}_{0}}{\bar{v}}=\Delta \tau_{\varphi}
$$

Figure 7 displays the behavior of the quantities $\Delta t_{T}$ and $\Delta t_{R}$ in function of $k$, for the physical parameters used in constructing Tables I and II. As a behavior limit, both tend to the phase time $\Delta \tau_{\varphi}$, where

$$
\Delta \tau_{\varphi}=\tau_{0} \frac{k_{0}^{2}(\sinh 2 \rho a / k \rho)-2 k a\left(k^{2}-\rho^{2}\right) / k_{0}^{2}}{\sinh ^{2} \rho a+\left(2 k \rho / k_{0}^{2}\right)},
$$

and $\tau_{0}$ is the characteristic time given by

$$
\tau_{0}=\frac{\hbar}{2 V_{0}}
$$

\section{NUMERICAL ANALYSIS}

In the following discussion and tables, $k$ is expressed in units of $k_{0}$, defined in Eq. (3). Lengths are expressed in units of the barrier width $a$. Generically, we assume $k_{0} a=1$, un-

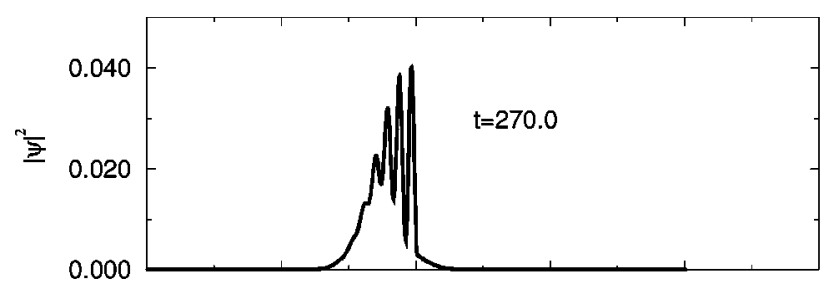

$\mathrm{x} / \mathrm{a}$
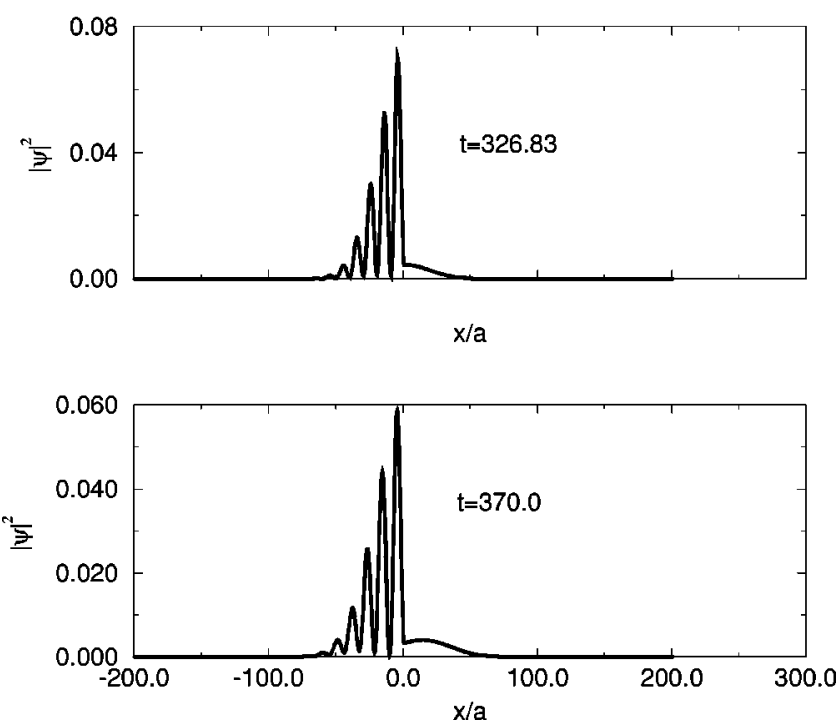

FIG. 8. Spatial distribution $|\psi(x, t)|^{2}$ for $t=220,326.83$, and 370 , in units of $\tau_{0}$.
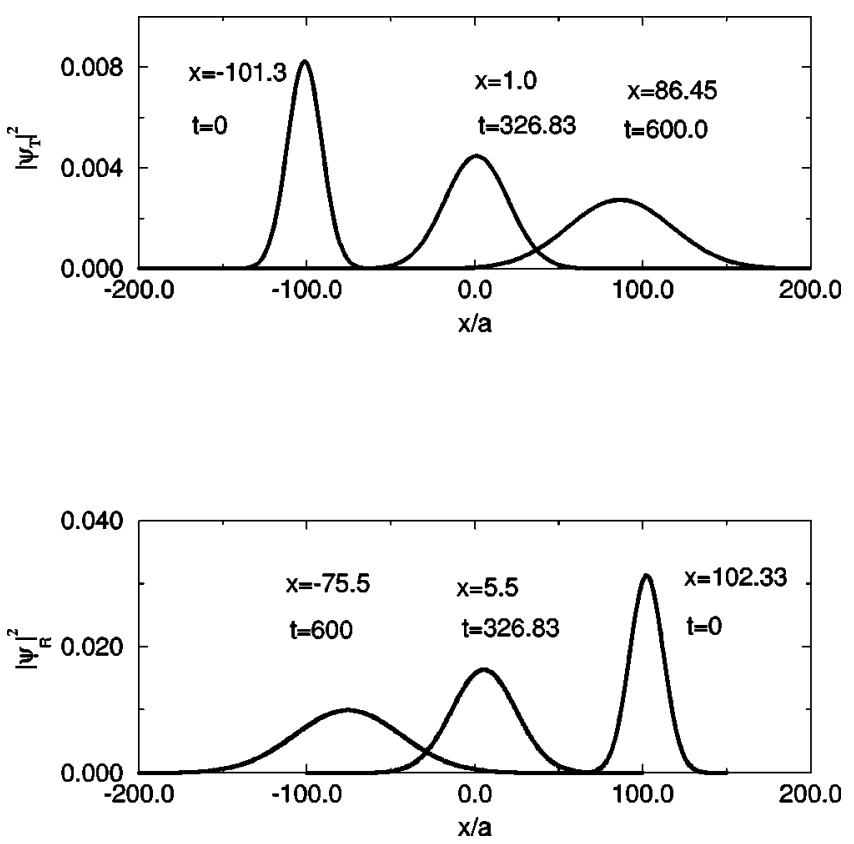

FIG. 9. Spatial distributions $\left|\psi_{R}(x, t)\right|^{2}$ and $\left|\psi_{T}(x, t)\right|^{2}$ for $t=0,326.83$, and 600 in units of $\tau_{0}$.

less otherwise explicitly pointed out. Times are given in units of the characteristic time $\tau_{0}$ defined in Eq. (55).

Let us consider the time evolution of the probability density function $|\psi(x, t)|^{2}$ associated with the amplitude distribution defined in Eq. (23). The initial distribution $|\psi(x, 0)|^{2}$ was constructed at $x_{0}=\ell=-100$. Figure 8 represents $|\psi(x, t)|^{2}$ for $t=220,326.83$, and 370 and a distribution $g(k-\bar{k})$ of width given by $\sigma_{k}^{2}=0.0025$, and centered in $\bar{k}$ $=0.3$. This figure shows us that the tunneling process is a very slow one.

Let us now consider $\psi_{I}(x, t), \psi_{R}(x, t)$, and $\psi_{T}(x, t)$ given by Eqs. (24), (26), and (34), respectively. A numerical analysis of the distribution $\left|\psi_{I}(x, t)\right|^{2}$, unnecessary in this case, would show that it propagates to the left with velocity $v$ $=\hbar \bar{k} / m$. The other two distributions, $\left|\psi_{R}\right|^{2}$ and $\left|\psi_{T}\right|^{2}$, are given in Fig. 9 for $t=0,326.83$, and 600. An analysis of the plottings in Fig. 9 allows us to associate with $\left|\psi_{R}(x, t)\right|^{2}$ a velocity $v_{R}=0.296 v_{0}$, and with $\left|\psi_{T}(x, t)\right|^{2}$ a velocity $v_{T}$ $=0.313 v_{0}$, where $v_{0}=\hbar k_{0} / \mathrm{m}$.

Notice that $\psi_{R}(x, t)$ and $\psi_{T}(x, t)$, taking into account their respective valid ranges $x<0$ and $x>a$, only contribute to the process after positive times $t_{R}$ and $t_{T}$ have elapsed.

TABLE III. Propagation velocity of the transmitted wave packet peak in function of the group velocity $\bar{v}=\hbar \bar{k} / m$ of the incident wave packet. $\Delta \tau_{T}$ is the transmission time. Comparison between the peak position $x_{T}$ and the expected value $\langle x\rangle_{T}$ is also shown, for $t=600$.

\begin{tabular}{cccccc}
\hline \hline $\bar{k}$ & $v_{T}$ & $\Delta \tau_{T}$ & $\Delta t_{T}$ & $x_{T}(t=600)$ & $\langle x\rangle(t=600)$ \\
\hline 0.30 & 0.313 & -6.33 & -6.23 & 86.45 & 86.59 \\
0.35 & 0.361 & -2.08 & -2.09 & 115.05 & 114.86 \\
0.50 & 0.506 & 1.58 & 1.59 & 202.60 & 202.63 \\
0.70 & 0.703 & 1.82 & 1.83 & 321.14 & 321.15 \\
\hline \hline
\end{tabular}


TABLE IV. Propagation velocity of the reflected wave-packet peak as a function of the group velocity $\bar{v}=\hbar \bar{k} / \mathrm{m}$ of the incident wave packet. $\Delta \tau_{R}$ is the reflection time. Comparison between the peak position $x_{R}$ and the expected value $\langle x\rangle_{R}$ is also shown for $t=600$.

\begin{tabular}{cccccc}
\hline \hline $\bar{k}$ & $v_{R}$ & $\Delta \tau_{R}$ & $\Delta t_{R}$ & $x_{R}(t=600)$ & $\langle x\rangle_{R}(t=600)$ \\
\hline 0.30 & 0.296 & 11.86 & 11.90 & -75.50 & -75.54 \\
0.35 & 0.346 & 9.72 & 9.77 & -105.38 & -105.39 \\
0.50 & 0.495 & 5.91 & 5.96 & -195.15 & -195.16 \\
0.70 & 0.695 & 3.56 & 3.56 & -315.15 & -315.17 \\
\hline \hline
\end{tabular}

In Tables III and IV, we display the group velocities of the transmitted and reflected packets, for some values of $\bar{k}$. The respective distributions have width $\sigma_{k}^{2}=0.0025$. The quantities $x_{T}$ and $x_{R}$ correspond to the maxima of the distributions determined from the plottings, and $\langle x\rangle_{T}$ and $\langle x\rangle_{R}$ were determined numerically and correspond to the expected values associated with those distributions at a given instant.

Transmission and reflection times. A numerical analysis of the time evolution of $\left|\psi_{T}(x, t)\right|^{2}$ allows us to associate with this distribution a velocity $v_{T}$, and a position $L_{T}\left(t_{T}\right)$ at a given instant $t_{T} \gg t_{0}$. With these data, an observer would associate to the process the expression (for particles in the region $x \gg a$ )

$$
t_{T}=\frac{\ell}{\bar{v}}+\Delta \tau_{T}+\frac{L_{T}\left(t_{T}\right)-a}{v_{T}},
$$

where $\ell / \bar{v}$ is the time a particle takes to reach the potential barrier, $\Delta \tau_{T}$ is the delay time associated with the transmission process, and $\left[L_{T}\left(t_{T}\right)-a\right] / v_{T}$ is the time taken by the particle to cover the distance $L_{T}\left(t_{T}\right)-a$ with velocity $v_{T}$.

From Eq. (56), we obtain

$$
\Delta \tau_{T}=t_{T}-\frac{\ell}{\bar{v}}-\frac{L_{T}\left(t_{T}\right)-a}{v_{T}} .
$$

A similar analysis for the reflection process leads to the following expression for the corresponding delay time:

$$
\Delta \tau_{R}=t_{R}-\frac{\ell}{\bar{v}}-\frac{L_{R}\left(t_{R}\right)}{v_{R}} .
$$

The numerical results obtained for $\Delta \tau_{T}$ and $\Delta \tau_{R}$, in comparison with the corresponding values $\Delta t_{T}$ and $\Delta t_{R}$ determined through the stationary phase method, for a given distribution $g(k-\bar{k})$ and some values of $\bar{k}$, are given in Tables III and IV.

\section{CONCLUSION}

In this section, we present some general conclusions and notes. For barriers of small width, $k_{0} a \ll 1$, and distributions $g(k-\bar{k})$ not centered around small $\bar{k}$, the reflection and transmission times both tend to the tunneling time $\Delta \tau_{\varphi}$ obtained through the method of phases.

Within some limits, to be discussed below, the values obtained for $\Delta t_{T}$ and $\Delta t_{R}$, for the given $\bar{k}_{T}$ and $\bar{k}_{R}$, are in complete agreement with a numerical analysis of the problem. The criticism of Büttiker and Landauer [3] that "the peak is not a reliable characteristic of packets distorted during the tunneling process" does not apply to the present analysis.

The limit of validity of the calculations carried out until now are directly connected to the approximate Gaussian form of the distributions $\phi_{T}(k)$ and $\phi_{R}(k)$. In Fig. 3, $\phi_{T}(k)$ has an approximate Gaussian shape for $2 \Delta k^{2}=0.01$ and $\bar{k}$ $=0.7$. In Hartmann's paper [2], such distributions around $\bar{k}$ $=0.7$ and $\Delta k=0.1 k_{0}$ are discarded. According to Hartmann it is necessary to introduce components with energies greater than the barrier height. The corresponding coefficients would be extremely small, as can be seen from his figure.

The numerical analysis carried out to obtain the time spent in the reflection and transmission processes shows that $\Delta t_{T}$ and $\Delta t_{R}$ obtained via stationary phase method are in excellent agreement with the numerical results. This kind of analysis, in the present case limited only by the approximate Gaussian form of the distributions $\left|\phi_{T, R}\right|^{2}$, does not present any difficulty when we deal with a distribution $g(k-\bar{k})$ of finite width $\sigma_{k}$, a concern of Hauge et al. pointed out by those authors in Ref. [4]. An analysis of the results obtained and presented in Fig. 7 shows the tendency of the quantities $\Delta t_{T}$ and $\Delta t_{R}$ to approach the phase time when $\bar{k} / k_{0} \rightarrow 1$. Additionally, for distributions $g(k-\bar{k})$ defined for $k$ in the vicinity of $\bar{k}$, i.e., $g(k-\bar{k}) \sim \delta(k-\bar{k})$, the quantities $\Delta t_{T}$ and $\Delta t_{R}$ approach $\Delta \tau_{\varphi}$. This quantity can be used as a very good approximation in these cases.

Another conclusion that can be drawn from the application of the stationary method to the analysis of the time evolution of a wave packet incident on a potential consists in the observation of a filtering effect due to the presence of the barrier. On average, particles traversing the barrier have velocity $\bar{v}_{T}$ such that $\left|\bar{v}_{T}\right|>|\bar{v}|$, and those reflecting have velocity $\bar{v}_{R}$ such that $\left|\bar{v}_{R}\right|<|\bar{v}|$.

The $\ell$ dependence of $\Delta t_{T}$ and $\Delta t_{R}$ is inherent in the process. It is related to the spreading of the wave packet as time flows.

As a last comment, in the case of a wave packet incident on a rectangular barrier with distribution $g(k)$ symmetric around $\bar{k}$, the application of the stationary phase method is limited only by the symmetric form of the distributions $\left|\phi_{T, R}\right|^{2}$. Our analysis, shown in Fig. 7 , is limited to the interval $0.3<\bar{k} / k_{0}<0.7$, since outside this interval the distributions $\left|\phi_{T, R}\right|^{2}$ tend to deform and the positions of the maxima $x_{T, R}(t)$ fail to coincide with the expected values $\langle x\rangle_{T, R}(t)$ associated with the distributions $\left|\phi_{T, R}\right|^{2}$. 
[1] D. Bohm, Quantum Theory (Prentice-Hall, New York, 1951).

[2] T. E. Hartman, J. Appl. Phys. 33, 3427 (1962).

[3] M. Büttiker and R. Landauer, Phys. Rev. Lett. 49, 1739 (1982).

[4] E. H. Hauge and J. A. Støvneng, Rev. Mod. Phys. 61, 917 (1989).
[5] E. H. Hauge, J. P. Falck, and T. A. Fjeldly, Phys. Rev. B 36, 4203 (1987).

[6] S. Collins, David Lowe, and J. R. Barker, J. Phys. C 20, 6213 (1987).

[7] A. Erdélyi, Asymptotic Expansions (Dover, New York, 1956).

[8] C. Cohen-Tannoudji, B. Diu, and F. Laloë, Mécanique Quantique (Hermann, Paris, 1973), Vol. 1. 